\title{
INFLUENCIA DE LOS RECURSOS Y LAS DINÁMICAS DEL TERRITORIO EN EL SOSTENIMIENTO DE LA AGROINDUSTRIA RURAL PANELERA DE SUPÍA, CALDAS
}

\author{
Carlos Julián Ramírez Gómez \\ Holmes Rodríguez Espinosa ${ }^{2}$
}

Recibido el 22 de agosto de 2015, aprobado el 23 de marzo de 2016 y actualizado el 19 de diciembre de 2016

DOI: 10.17151/luaz.2017.44.12

\section{RESUMEN}

Después del café, la panela constituye la segunda agroindustria rural más importante en Colombia. El subsector panelero presenta actualmente problemas relacionados con bajos niveles tecnológicos y estándares de calidad, débil cultura organizativa y asociativa y acceso precario a mercados; retos que implican la necesidad de buscar estrategias desde los territorios en aras de buscar fortalecer el subsector. El estudio tuvo por objetivo analizar la influencia de los recursos y las dinámicas del territorio con el fin de determinar cómo se configura la generación de estrategias locales para la supervivencia y el sostenimiento de la producción panelera en Supía, Caldas, Colombia. Se realizó un estudio descriptivo con enfoque metodológico cualitativo y recolección de los datos en tres fases: estudio exploratorio, entrevistas semiestructuradas con actores del sistema agroalimentario localizado (SIAL), utilizando la técnica de muestreo de bola de nieve, talleres participativos con grupos de productores, observación y diario de campo. Finalmente se adelantó una fase de análisis y triangulación de la información, enmarcada en las dimensiones: histórica, técnica, institucional y alimenticia. Se encontró que las transformaciones ocurridas en los últimos 50 años, dan cuenta de la existencia de un SIAL dinámico con anclaje territorial que se soporta en recursos locales tales como el saber-hacer, las condiciones locales de clima y suelo y procesos de innovación tecnológica y organizacional, lo que ha permitido generar una reputación. Se identificaron en el territorio cinco tipos de agroindustrias rurales (AIR), algunas de las cuales han logrado transformar los recursos del territorio en activos territoriales, a través de dinámicas como las acciones colectivas estructurales o familiares, y diversas formas de relacionamiento tanto dentro de las propias AIR así como con otros actores del territorio, lo cual les ha permitido posibilidades de comercialización, entre otras ventajas que han contribuido al sostenimiento de la actividad panelera.

\section{PALABRAS CLAVE}

Acción colectiva, asociatividad, sistema agroalimentario localizado.

Influence of resources and dynamics of the territory in sustaining raw sugar rural agribusiness at Supía, Caldas

\begin{abstract}
After coffee, panela is the second largest, rural agribusiness in Colombia. The panela subsector currently presents problems related to low technological levels and quality standards, weak organizational and associative culture as well as markets' access. These challenges imply the need to seek strategies from the territories in order to strengthen the subsector. The aim of this research was to analyze the influence of
\end{abstract}


resources and the dynamics of the territory, in order to determine how to generate local strategies for survival and sustaining raw sugar production in Supía, Caldas, Colombia. A descriptive study with a qualitative methodological approach with data collection in three phases: an exploratory study, interviews with actors of localized agro-alimentary system (SIAL), using the technique of snowball sampling, participatory workshop and field diary, was conducted. An analysis and triangulation of information analyzing dimensions: historical, technical, institutional and food, was carried out. It was found that technical, social and economic changes over the last 50 years, account for the existence of a dynamic, local food system with know-how that constitutes one of the most important resources in the region; as well as technology, local conditions of climate and soil and innovation processes, which has generated a reputation. Five types of rural agribusiness (AIR) were identified. Some of them have been able to transform the region's resources into territorial assets, through structural or familiar collective actions as well as several relationships both within the AIR itself as a territorial level, which has played a key for sustaining panela activity, by improving their access to market.

\section{KEYWORDS:}

Collective action, associativity, localized agro-alimentary systems.

\section{INTRODUCCIÓN}

La panela en Colombia es la segunda agroindustria rural más importante después del café, ocupa $12 \%$ de la población rural económicamente activa y es reconocida como un caso representativo en América Latina por la diversificación de empleo y de ingresos para los pequeños productores campesinos, pues se estima que existen cerca de 70.000 familias de los andes colombianos que cultivan la caña panelera y 20.000 trapiches en los que se elabora panela y miel de caña, generando anualmente más de 2,5 millones de jornales y alrededor de 353.366 empleos directos (MADR, 2006; Martínez, Ortiz, \& Acevedo, 2005).

En un estudio efectuado por el Ministerio de Agricultura y Desarrollo Rural y la Corporación Colombia Internacional (2012) en los seis departamentos más relevantes en Colombia frente a la producción panelera, estableció la existencia de 11.472 trapiches, excluyendo grandes productores del país. Adicionalmente, los precios pagados a los productores han fluctuado entre $\$ 1.375$ a $\$ 1.701$ por kilogramo producido de panela.

El subsector panelero constituye un complejo productivo y agroindustrial en el cual se identifican diversas problemáticas nacionales entre las cuales se encuentran la deficiente tecnología de cultivo y transformación que afectan la calidad del producto; la estacionalidad de la producción y la competencia con el azúcar como producto sustituto que inciden en los precios de venta; débil cultura de la asociatividad y baja capacidad de articulación de actores que afectan la organización gremial (Castellanos, Torres, \& Flórez, 2010).

Dichas problemáticas del subsector, han buscado resolverse desde un enfoque de cadena productiva, por medio de la identificación de variables tecnológicas y no tecnológicas que inciden en el desempeño del subsector panelero y el planteamiento de diversas acciones por implementar a partir de acuerdos nacionales de fortalecimiento de la competitividad (MADR, 2001). 
Sin embargo, la agroindustria panelera se lleva a cabo bajo variadas condiciones locales en las cuales las interrelaciones tejidas por los diversos agentes sociales y económicos son heterogéneas en cada región (Rudas \& Forero, 1995); en consecuencia, las particularidades y situaciones diferenciales de cada territorio no han sido elementos considerados en las propuestas de fortalecimiento de la cadena productiva.

En ese sentido, entra en contexto la competitividad desde el territorio, lo cual implica el aprovechamiento de sus recursos endógenos, por lo cual se requiere estimular la capacidad de detectar las potencialidades propias, naturales, humanas, institucionales y organizacionales presentes (Silva, 2003).

Es así como surge el concepto de sistema agroalimentario localizado (SIAL), definido como una red compleja de relaciones entre las personas, los productos y los territorios, a través de un espacio construido socialmente y regulado institucionalmente (Boucher, 2001); constituido por sus organizaciones de producción, de servicio, unidades agrícolas, empresas agroalimentarias, empresas comerciales, entre otras (Muchnick, 2004).

De esta manera, el concepto de SIAL permite abordar la relación y vínculos existentes entre las actividades relacionadas por parte de las concentraciones de agroindustrias rurales (AIR) y el territorio, así como los efectos de estos en la actividad económica local y regional (Boucher, 2013). Adicionalmente, el enfoque SIAL se ha constituido en un marco analítico de los procesos de entendimiento de los recursos locales y del anclaje territorial de las producciones agrícolas y agroalimentarias (Fournier \& Muchnik, 2012).

Por ende, un SIAL se compone de una AIR, un territorio y la acción colectiva; la AIR es una concentración geográfica de pequeñas empresas rurales que generan transformación y valor agregado a los productos, con ventajas que suelen permanecer pasivas, pero en diversos casos cuentan con capacidades intrínsecas que permiten generar perspectivas de desarrollo (Boucher, 2013).

Estas organizaciones productivas constituyen concentraciones localizadas que presentan diversas características y funcionamientos, por lo cual deben analizarse su visión funcional y su rol dentro del sistema agroalimentario.

Por su parte, la acción colectiva es definida por Boucher (2006), como aquellos relacionamientos y vínculos territoriales, que se generan como el resultado de la convergencia de estrategias e intereses de un grupo de actores con intereses comunes. Entre tanto, la acción colectiva puede ser de carácter estructural, a partir de un grupo organizado y funcional, con un conjunto de normas, redes sociales, formas y relaciones de confianza que determinan comportamientos, entre otras (Boucher, 2006; Correa, Boucher, \& Requier-Desjardins, 2006; Ramos \&Salcido, 2011).

Adicionalmente, otros autores plantean que la acción colectiva está ligada a las relaciones entre los actores y la coordinación horizontal entre ellos, en donde la cooperación existente entre el grupo de actores, les puede ofrecer ventajas que sería difícil de conseguir de forma aislada, permitiendo generar estrategias conjuntas de competitividad (Pomeón, Boucher, Cervantes, \& Fournier, 2006; Salas, Boucher, \& Requier-Desjardins, 2005).

Por lo anterior, algunos autores como Salas et al (2005), plantean que en algunas AIR se genera una acción colectiva en torno a la construcción de activos específicos, 
impactando en las capacidades de pequeños productores, por ejemplo para lograr una inserción más eficiente en las cadenas de producción (Boucher, 2006). De hecho, muchas de las dinámicas de las AIR tienen correspondencia con los relacionamientos hacia adelante y hacia atrás en la cadena productiva, es decir, la interacción, las formas de coordinación de actores en un territorio local y la consolidación de redes de actores, también son aspectos destacados en torno a la AIR, en el marco del sistema agroalimentario (Boucher \& Reyes, 2011).

Por su parte, el territorio está conformado por recursos específicos, propios y disponibles en la región para las actividades productivas, que pueden ser transformados en activos, dependiendo de las formas de relacionamiento dentro del territorio, configurando una actividad agroalimentaria con ventajas competitivas (Bello, 2009; Boucher, 2006; Rodríguez-Borray \& Rangel-Moreno, 2005).

Adicionalmente, el territorio puede contar con un conjunto de recursos territoriales locales, tales como el saber hacer, la tecnología, la reputación del producto, condiciones locales de clima y suelo, diferentes formas de proximidades y procesos de innovación, tecnológica u organizacional, entre otros, los cuales se vinculan con dinámicas de desarrollo local y agroalimentario (Pomeón \& Fraire, 2011).

El enfoque SIAL tiene en cuenta el territorio como un actor estratégico en la competitividad de las actividades productivas locales, lo que proporciona una visión diferenciadora e incluyente respecto de dinámicas y recursos locales que son activados y empleados en la construcción de alternativas, y generan una diferenciación con el tradicional enfoque de cadena productiva con sus actores directos, indirectos y su funcionamiento fundamentalmente vertical (Specht, 2009).

Por lo tanto, el análisis de SIAL debe considerar los recursos locales del territorio, las personas, sus instituciones, su saber hacer y sus redes de relaciones (Muchnik, 2006); en el cual los procesos de acumulación, innovación y formación de capital humano y social tienen un carácter localizado (Moncayo, 2003).

EI SIAL tiene en cuenta un enfoque horizontal de la cadena productiva, delimitado por relaciones innovadoras hacia un mejor entendimiento entre actores, donde predominan el aprendizaje, la flexibilidad, la confianza y la diversidad (Murdoch, 2000); permitiendo que los pequeños productores aprovechen los recursos locales del territorio, activados a través de acciones colectivas, para el desarrollo de potencialidades y generación de ventajas competitivas (Correa et al., 2006; Sandoval \& Ruiz, 2005); al igual que relaciones con las instituciones, los proveedores de insumos y los servicios de apoyo (Sandoval, 2003).

En diversos territorios paneleros colombianos, ciertas características locales han permitido generar mecanismos de respuesta a los desafíos del sector, lo cual ha facilitado el sostenimiento de la producción de manera localizada y a lo largo del tiempo; permitiendo la identificación de cerca de 15 SIAL paneleros, con diversas escalas productivas y niveles tecnológicos, con sus propias especificidades agroecológicas, socioeconómicas, recursos locales, instituciones y actores con un rol determinado (Rodríguez, García, Roa, \& Santacoloma, 2004).

En consecuencia, el presente estudio tuvo por objetivo analizar la influencia de los recursos y las dinámicas del territorio mediante herramientas cualitativas con el fin de determinar cómo se configura la generación de estrategias locales para la supervivencia y el sostenimiento de la producción panelera en el municipio de Supía, Caldas. 


\section{MATERIALES Y MÉTODOS}

Se realizó un estudio descriptivo con enfoque metodológico cualitativo, de carácter interpretativo (Denzin \& Lincoln, 1994; Lamnek, 2005; Noelle-Neumann \& Petersen, 2005), utilizando la técnica de estudio de caso (Simons, 2011; Yin, 2013), por medio de la cual se realizó una evaluación completa de los acontecimientos en un marco geográfico definido en un período determinado, buscando comprender en profundidad el fenómeno específico del territorio panelero, por medio de la observación, registro y análisis de interacciones entre personas y entre éstas y sistemas (Cunha, 2012).

Para el desarrollo de la investigación se llevaron a cabo tres fases de la siguiente manera: estudio exploratorio, entrevistas con actores del SIAL, análisis y triangulación de la información.

\section{Fase 1: Estudio exploratorio}

Inicialmente se realizó un estudio exploratorio en el cual se entrevistó, mediante herramienta semiestructurada, un proveedor de insumos importante de la región, una persona de la institucionalidad administrativa municipal y el líder de la cooperativa de productores paneleros "Coimpas". Las preguntas del guión tenían el propósito de identificar principales veredas productivas, líderes y actores claves en las veredas, posibilidades y formas de contactos, veredas con innovaciones en productos, generalidades y la dinámica agroalimentaria del territorio panelero.

\section{Fase 2: Entrevistas con actores del SIAL}

Una vez adelantada la fase anterior se procedió al levantamiento de la información a partir de la aplicación de 23 entrevistas semi-estructuradas en el período comprendido entre enero de 2013 y enero de 2014, con productores en 5 veredas representativas del municipio: Mudarra, Alto Sevilla, San Pablo, Guascal y Dosquebradas. De igual manera se efectuaron dos entrevistas con el líder de la cooperativa de productores paneleros de Supía (Coimpas) y la profesional de la Federación Nacional de Productores de Panela (Fedepanela) en el municipio.

Para la selección de los productores entrevistados en las veredas de estudio, se procuró que tuvieran la capacidad de hacer una retrospectiva territorial histórica de 50 años y que tuvieran una relación directa con la producción panelera. Para ello se utilizó la técnica de bola de nieve, consistente en seleccionar un informante clave a criterio del investigador, al cual se le pide que nomine o sugiera a otras personas o actores dentro de la población objetivo (Martín-Crespo \& Salamanca Castro, 2007), proceso que se repite hasta que el investigador determine que no hay información adicional por recolectar

Adicionalmente, las preguntas contenidas en la entrevista fueron planteadas y adaptadas a partir de la metodología de estudio del enfoque SIAL propuesta por Muchnik (2006), en la cual se propone un abordaje multidimensional del sistema agroalimentario, buscando profundidad histórica para identificar la evolución del SIAL en el tiempo, así como la identificación de recursos y dinámicas del territorio (Tabla 1). 
Tabla 1. Dimensiones de estudio para el análisis del SIAL

\begin{tabular}{|c|c|}
\hline Dimensión & Características \\
\hline Histórica & $\begin{array}{l}\text { Proporciona información para la interpretación de transfomaciones ocurridas en } \\
\text { el desarrollo del sistema agroalimentario con una visión retrospectiva en los } \\
\text { últimos } 50 \text { años. }\end{array}$ \\
\hline Técnica & $\begin{array}{l}\text { Permite el análisis del saber-hacer empleado en el SIAL, bien sea en el cultivo o } \\
\text { la transformación de un producto agroalimentario, por lo alal se interpreta } \\
\text { también porqué podría considerarse un SIAL diferenciado. }\end{array}$ \\
\hline Institucional & $\begin{array}{l}\text { Se analizan el entendimiento y las relaciones tanto en la agroindustria rural } \\
\text { como de los actores del SIAL, así como los vínculos basados en sus estrategias } \\
\text { individuales o colectivas entre los actores del sistema agroalimentario local. }\end{array}$ \\
\hline Alimenticia & $\begin{array}{l}\text { Se analiza qué características pemiten que el producto pueda ser diferenciado } \\
\text { y con un valor agregado desde el territorio o la agroindustria rural. Así mismo se } \\
\text { tiene en cuenta la procedencia de los productos y la confianza generada con el } \\
\text { consumidor. }\end{array}$ \\
\hline
\end{tabular}

Fuente: Los autores con base en Muchnik (2006).

Fase 3: Análisis y triangulación de la información

Se procesó la información a través del establecimiento de variables que permitían sustentar las categorías de análisis del enfoque SIAL, con el fin de profundizar y entender la dinámica productiva y las interacciones tanto en la agroindustria rural como en el territorio, así como el rol que desempeñan recursos y dinámicas del territorio acorde con el objeto de estudio.

Por otro lado, para el análisis y triangulación de la información también se tuvo en cuenta la noción de estructura del sistema agroalimentario localizado, propuesta por Boucher \& Reyes (2011), en la cual se clasificaron las agroindustrias rurales, y la acción colectiva como una de las dinámicas del territorio y que se enmarcaron posteriormente en la dimensión institucional para su análisis. Para tal efecto también se desarrolló un diario de campo donde se registró la observación directamente, la cual fue luego complementada con información secundaria.

Finalmente, una vez clasificadas las agroindustrias rurales, se procedió a complementar el estudio y las dimensiones de análisis con un abordaje a partir de redes sociales, el cual se llevó a cabo empleando la metodología cualitativa Net Map(Schiffer, 2007), que consiste en una entrevista con pequeños grupos de productores. La metodología sirve como una herramienta que ayuda al investigador y a las personas abordadas a entender, visualizar, analizar, discutir y evaluar cómo diferentes actores pueden influir o intervenir frente a alguna situación particular o a un sector.

El mapeo de la red es el resultado de una discusión y análisis con el grupo de productores y se construye a partir de un taller participativo con las personas del grupo. Así mismo, este mapeo de la red permitió representar actores involucrados en la producción panelera, así como identificar las interrelaciones y su influencia en el 
subsector panelero. Para graficar la red, las entrevistas grupales se enfocaron en el análisis del flujo de información, la cooperación y la confianza.

Esta herramienta permitió aproximarse al entendimiento de la red social panelera que configura el SIAL, visualizando la gráfica como un reflejo de la estructura social, vista desde la perspectiva de la red en el ámbito local.

\section{RESULTADOS}

\section{El territorio panelero de Supía}

El municipio de Supía implica la conformación de un territorio con recursos que combina elementos biofísicos, socioeconómicos así como culturales y que permiten dimensionar el contexto en el cual se desarrolla propiamente en este caso la actividad panelera. Entre tanto, esta contextualización permite comprender cómo este territorio ha configurado un sistema agroalimentario que se ha soportado en recursos, activos específicos y la influencia de diversas dinámicas sociales.

El territorio panelero está conformado por una gran riqueza étnica, en la cual se destacan comunidades campesinas mestizas, afrodescendientes e indígenas. Esta presencia intercultural ha generado intercambios y consolidado una cultura a lo largo de la historia, a partir de medios de vida, de las formas de ordenamiento entre los individuos y su entorno, fortalecimiento de los lazos de comunicación y de saberes que se han instaurado en el territorio y que se han transmitido a lo largo de las generaciones (Jurado \& Botero, 2012).

La producción primaria es fundamentalmente artesanal y a pequeña escala, con fincas que pueden variar entre 1 a 3 hectáreas. Se encuentra conformada por cerca de 480 trapiches con 1300 productores integrados por grupos familiares y comunitarios, inscritos a organizaciones con reconocimiento jurídico; el resto, hace parte de los grupos del mismo orden, sin tal condición (Jurado \& Botero, 2012).

Del mismo modo, el proceso de transformación a panela implica un conglomerado de pequeñas agroindustrias rurales paneleras que desarrollan diversas relaciones y acciones colectivas que constituyen dinámicas del territorio panelero de Supía, a partir de un esquema de producción relativamente artesanal que ha venido modificando paulatinamente su esquema de tecnificación (Corrales \& Forero, 2007; Gómez \& Buitrago, 2008).

\section{Dimensión histórica: transformaciones del territorio en los últimos 50 años}

La producción panelera de Supía se ha llevado a cabo tradicionalmente por familias campesinas de diferentes veredas con una evolución a lo largo del tiempo en la ocupación del territorio y transformaciones técnicas, económicas y socio-culturales (Figura 1). Pese a ser un territorio de gran influencia minera y de predominio cafetero en partes más altas, la crisis de los ochenta dio lugar a una gran expansión productiva de la caña en el territorio. No obstante, la cultura panelera ha configurado con anterioridad una identidad local a partir de las fiestas de la colación, que se arraigaron desde finales de los años 50 e inicios de la década de los sesenta (IICA, n.d.). 


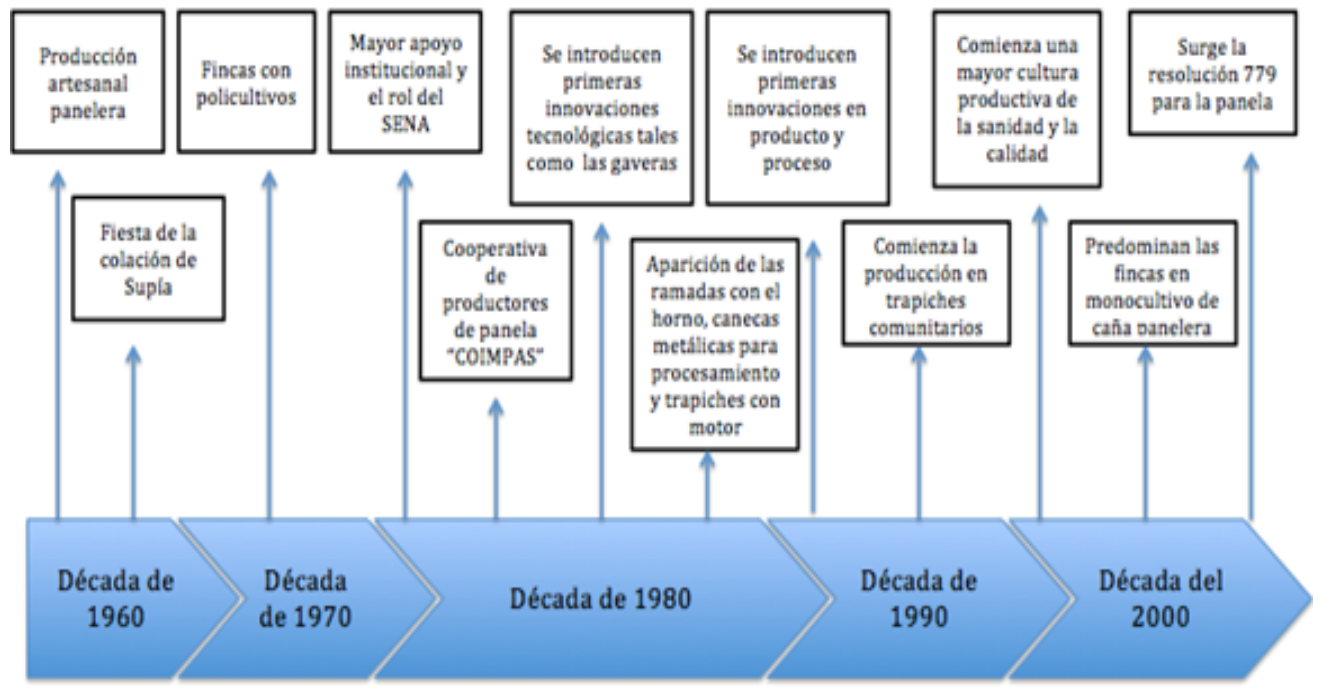

Fuente: Los autores, 2015

Figura 1. Línea de tiempo de la evolución panelera de Supía.

Los cambios técnicos generados en el subsector panelero de Supía, vienen originándose al menos en los últimos 50 años, no obstante el sistema de producción artesanal aún subsiste, a través de un modelo tradicional sin uso de abonos químicos, plaguicidas, formas de corte por desguíe, entre otros. Hacia los años 80, inicia un apoyo institucional donde se destaca el rol del SENA, que coadyuvó con la introducción de las primeras innovaciones tecnológicas, en particular en la presentación, trascendiendo de la panela de $500 \mathrm{gr}$ comúnmente llamada "casco de buey", a la diversificación a través del surgimiento de moldes de 125 y 150 gr llamados "gaveras", lo cual fue mejorando el posicionamiento en diversos mercados.

Finalmente, hacia los años 90 se introduce una de las transformaciones técnicas más importantes y tiene relación con innovaciones en proceso y producto, pues aparte de la panela en pastilla y en cuartos, surge la panela pulverizada, cuando el mercado comenzaba a exigir nuevas formas de presentación y el empaque, el cual ha evolucionado desde los años 70 y hasta la actualidad. Adicionalmente, las transformaciones en la agroindustria rural panelera han conducido al mejoramiento de la infraestructura, empaques y rendimientos.

Por su parte, las agroindustrias rurales fueron sufriendo transformaciones sociales que implicaron una adaptación a una nueva cultura de cambio técnico, donde la noción de calidad ha venido arraigándose. A partir de los años 90 , las prácticas se comienzan a desarrollar con mayor conciencia de la producción de un alimento y solo a partir del año 2000 , los productores prestan mayor atención al asunto de la sanidad y la calidad, arraigando esta cultura.

Adicionalmente, después de la crisis cafetera de los años 80 y la mayor expansión de la producción panelera, entran con mayor fuerza los trapiches comunitarios, propiciando en general una cultura de la cooperación y solidaridad en el territorio, motivados por la Resolución 779 de 2000, supeditando al campesinado a la asociatividad en aras de mejorar su proceso de calidad, pero también de sostenibilidad en los mercados. Sin embargo, cabe destacar que un factor limitante en el proceso de cooperación y asociatividad tiene relación con las formas de producción de la caña, y la diversidad de sus calidades, asunto que afecta en muchos casos los entendimientos entre productores en aras de la calidad del jugo y su transformación a panela. 
El territorio panelero de Supía evidencia a lo largo de su historia transformaciones económicas, no solo a partir de la ocupación del espacio en las fincas, sino también por la rentabilidad y la forma en que esta se ha venido presentando. Es preciso mencionar que en los últimos 50 años, las fincas que antes eran dedicadas a policultivos, en la actualidad se dedican a la producción de caña como monocultivo. Aunque el trabajo comunitario y el mejoramiento tecnológico ha hecho más rentable la actividad panelera, también es cierto que la ausencia de producción de alimentos en los predios aumenta el gasto en alimentación.

En todo caso diferentes transformaciones técnicas, sociales y económicas de la actividad panelera en Supía, dan cuenta de cambios cimentados por un contexto histórico, generados en los últimos 50 años e influenciados por diversos aspectos, por lo cual fue necesario un acercamiento con las comunidades locales.

\section{Dimensión técnica: análisis del saber-hacer utilizado en el SIAL}

Los saberes y técnicas han generado un conocimiento que se ha transmitido de una generación a otra por más de 50 años en la región, constituyendo prácticas y creencias ancestrales. Diversos conocimientos tácitos han sido obtenidos por la experimentación propia de los campesinos arraigando un saber hacer en búsqueda de la calidad. De esta manera, las formas de siembra según calendario lunar, la forma de corte llamado por desguíe y una cultura de la producción que en términos generales tiene arraigada la visión de calidad y procesamiento más limpio de la panela, busca el reconocimiento y la diferenciación de su producto desde el territorio.

Por otro lado, la tecnología constituye otro recurso territorial destacado, expresado en maquinaria, infraestructura de trapiches y prácticas tendientes a la higiene, mantenimiento y aseo de trapiches. El uso de gorras, camisa, tapabocas, al igual que el colado de jugos, han incidido en el mejoramiento de la producción.

Adicionalmente, diversos tipos de vínculos, formas de asociación y cooperación y estrategias tanto individuales como colectivas de los productores, han consolidado un recurso del territorio para el sostenimiento de la actividad panelera en la región. Este recurso también implica el apoyo institucional recibido a través de diversos tipos de recursos y sus relacionamientos locales. También se destacan diversas formas de arreglos de ciertos trapiches comunitarios, que permiten soportar la confianza, la corresponsabilidad, el aprendizaje colectivo y los acuerdos que facilitan entendimientos, al mejorar la competitividad y el acceso a mercados, así como la transmisión del saber hacer y el conocimiento.

Así mismo, existen diversas formas de agroindustrias rurales representadas en trapiches paneleros, donde a partir del saber hacer se produce la panela con diferentes presentaciones como redonda, cuadrada o pulverizada; incluso es común el valor agregado generado por pequeños productores en la fabricación de dulcerías, como blanqueados, colaciones, entre otros (Gómez y Buitrago, 2008). Por último, la producción suele adelantarse generalmente aún de manera artesanal.

\section{Dimensión institucional: relaciones de los actores del SIAL}

EI SIAL panelero de Supía está integrado por una concentración geográfica de agroindustrias rurales que se apoyan en los recursos territoriales y en las dinámicas del territorio que implican diversas formas de acción colectiva, formas de entendimiento y vínculos, tanto dentro de las AIR así como externos, con otros actores del territorio y articulaciones a las cadenas de producción. Estos procesos se han 
generado a partir de varias características que diferencian cinco tipos de trapiches paneleros, con base en su estructura de conformación, acuerdos en materia de calidad, manejo de materia prima y procesamiento y sus características de organización e incursión en los mercados: AIR particular, AIR individual artesanal, AIR familiar artesanal, AIR comunitaria asociada y AIR comunitaria no asociada (Tabla 2).

\section{AIR particular}

Esta agroindustria rural se destaca por la modernidad de sus equipos e instalaciones. Sus altos volúmenes de producción y las condiciones agroecológicas favorables del territorio para la actividad panelera, les permiten también combinar criterios de calidad, lo cual le permite acceder a unos mercados directos que logran excluir en muchos casos a la intermediación en la cadena de comercialización. Sin embargo, no es un modelo predominante en Supía.

Tabla 2. Clasificación y características de las agroindustrias rurales paneleras de Supía.

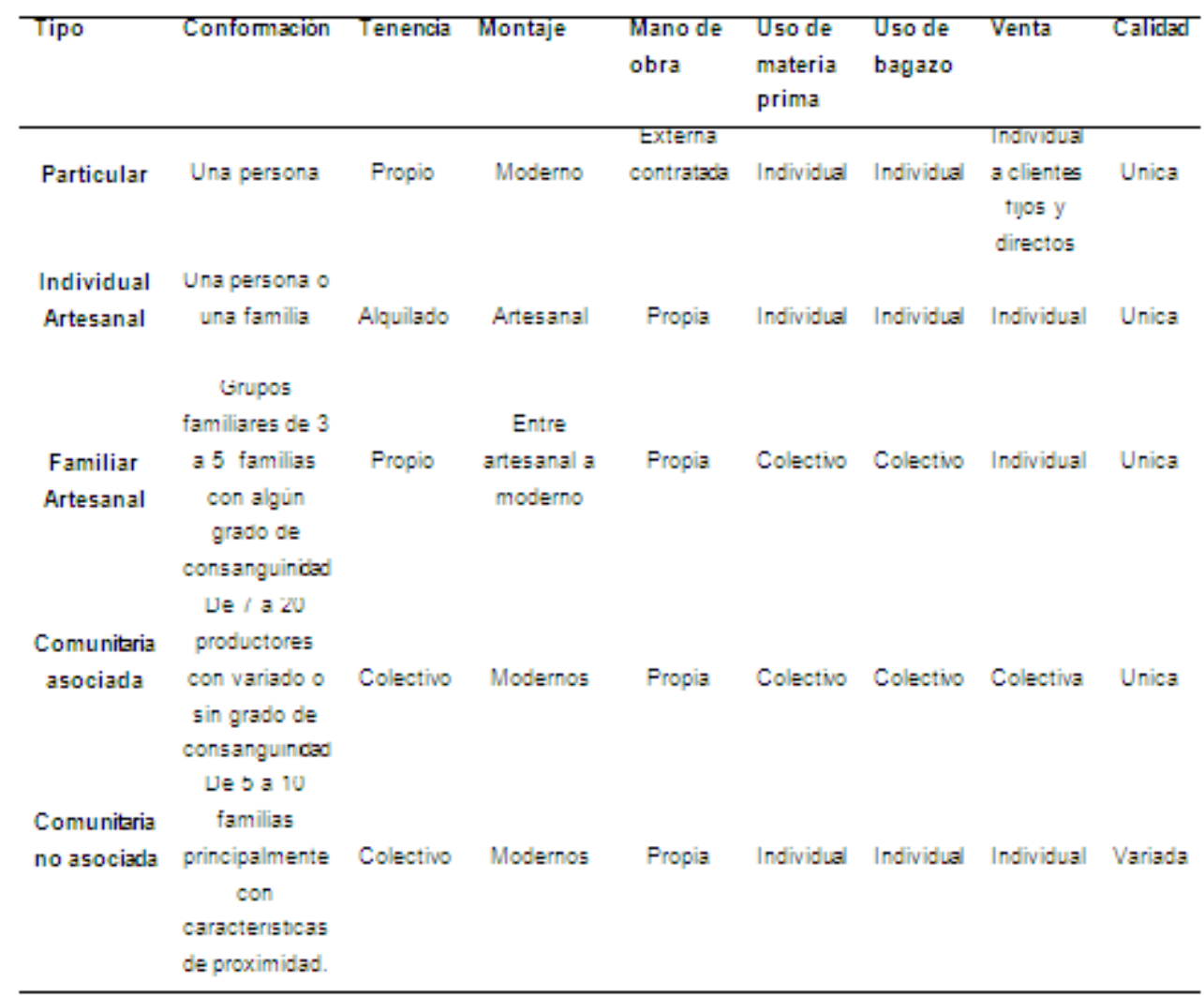

Fuente: Los autores, 2015.

\section{AIR individual artesanal}

Otro tipo de organización productiva es la AIR individual artesanal, la cual se basa fundamentalmente en molinos de tracción animal a través del empleo de caballos y mulas, o accionados por pequeños motores (Zuluaga, 2001). Este tipo de AIR está constituido a través de relacionamientos de un dueño con vecinos o amigos que no cuentan con montaje para producción, para lo cual este suele ser alquilado para la labor de molienda y fabricación. 
Esta AIR funciona de manera individual sin acción colectiva estructural; sino basada en relaciones de amistad, donde cada persona y su núcleo de apoyo que podría ser o no familiar, procesan en su día acordado con el dueño del trapiche, quien actúa solo como proveedor de la infraestructura. Como normas y reglas, cada persona podría pagar algún monto por el alquiler bien sea representado en dinero o en porcentaje de la producción. No obstante, la persona que acude a procesar debe llevar sus insumos correspondientes, así como entregar aseado el montaje.

Tiene bajos niveles de innovaciones en el producto y dificultades de inserción al mercado por cuanto producen tantas calidades de panela como personas y su núcleo de apoyo utilicen el trapiche. La comercialización es individual, sin niveles de cooperación colectiva en venta a partir de la oferta de mayores volúmenes.

\section{AIR familiar artesanal}

Por otro lado, la AIR familiar artesanal desarrolla su producción panelera dentro de un esquema de economía campesina, generalmente en trapiches de tracción mecánica con una capacidad de 50 a $100 \mathrm{~kg} /$ panela/hora. En cuanto a lo organizacional, esta AIR soporta su organización en relacionamientos familiares y en la proximidad de sus miembros, donde se destaca el accionar colectivo entre todos los miembros del trapiche, con cooperación e intercambio entre las familias, de recursos tangibles y no monetarios como precios y canales de venta. Pese a todo esto, esta organización se ve sometida al mercado intermediario aunque produzcan una sola calidad de panela.

Existen relaciones horizontales reflejadas en la confianza, el aprendizaje mutuo y reglas consensuadas. Pactan días de trabajo y molienda y acuden todos como fuerza laboral al procesamiento. No existen conflictos por las calidades de la materia prima ni por el uso del bagazo necesario en el proceso. Otras actividades posteriores al procesamiento, tales como el aseo de la instalación, las realizan de manera colectiva y procuran aplicar buenas prácticas de manufactura en muchos de los casos.

\section{AIR comunitaria asociada}

Otra clase de trapiche panelero existente en Supía es la AIR comunitaria asociada. Este tipo de organización productiva de la panela ha venido cobrando mayor importancia por la relevancia que viene teniendo la asociatividad en el territorio rural, a partir por ejemplo de una mayor posibilidad de acceso a recursos y programas de apoyo departamental y nacional, en particular para cumplir con la Resolución 779 de la panela que implica una inversión en instalaciones, con el fin de implementar buenas prácticas de manufactura para la producción panelera, orientadas a garantizar al consumidor la calidad e inocuidad del producto; las cuales además, pueden favorecer la inserción de estos productores en mercados especializados, supermercados y tiendas de gran distribución.

Algunos trapiches pueden emplear motores de energía eléctrica, a gasolina y diésel y con capacidades de procesamiento que oscilan entre 150 y $900 \mathrm{~kg} /$ caña/hora (Gómez \& Buitrago, 2008). Es común que se asignen roles específicos de acuerdo con las capacidades de cada socio para tener mayor eficiencia en el procesamiento, lo cual da cuenta de relacionamientos ampliamente horizontales entre sus miembros, donde predomina la confianza, el entendimiento y aprendizaje colectivo y hasta generación de estatutos de la organización.

Estas organizaciones producen una sola calidad de panela lo cual les facilita generar un núcleo de poder frente al eslabón de mercado, en presentación de $500 \mathrm{gr}$ y $250 \mathrm{gr}$ redonda o cuadrada. Así mismo, esta agroindustria rural se incorpora a redes 
territoriales en sus mejores relacionamientos con actores locales o externos. Entre tanto, presenta una acción colectiva estructural, con volúmenes de producción, calidad e inserción a mercados, teniendo muchas veces núcleo de poder frente a negociaciones con comercializadores.

En este grupo existen AIR constituidas legalmente y registradas en Cámara de Comercio y otras bajo la formalidad que ellos han generado a partir de sus propias instituciones, mas no conformados como figura empresarial formal. No obstante, en ambos esquemas se hacen reuniones, relacionamientos horizontales, existencia de un fondo rotatorio de la organización y reglas de funcionamiento y se asume un papel altamente colectivo de los miembros de la AIR.

\section{AIR comunitaria no asociada}

La última clase de trapiche panelero se denomina AIR comunitaria no asociada, muy común principalmente en zonas donde existen las veredas con mayor actividad panelera del municipio, en las cuales en aras de buscar cumplir con la normatividad 779 , algunas instituciones gubernamentales han donado trapiches con instalaciones adecuadas, en veredas específicas, donde un grupo de personas deben tratar de juntarse para fines de producción y uso de la infraestructura.

Su tecnología también tiene similitud con algunos trapiches comunitarios asociados, donde se pueden emplear motores de energía eléctrica, a gasolina y diésel y con capacidades de procesamiento que oscilan entre 150 y 900 kg/caña/hora (Gómez \& Buitrago, 2008). Mantienen unas mínimas reglas como reuniones quincenales, cuotas de manejo y compromisos de mantenimiento de instalación y equipos.

En este caso, este tipo de organización usualmente no logra acuerdos entre sus miembros en materia de calidad de la materia prima y de la panela procesada, es decir, no existe una sola calidad de panela, sino tantas calidades como familias que producen en el trapiche, lo cual significa que sus miembros comercializan por separado su producción, perdiéndose la posibilidad de competir con calidad y volúmenes, así como de la oportunidad de asociarse para acceder a convocatorias públicas de fortalecimiento de organizaciones empresariales rurales, vínculos con diversas entidades, entre otras.

De igual manera suelen desconocerse las formas de producción de la panela entre sus mismos miembros socios del trapiche. Aunque se busca el entendimiento de las familias socias, las cuales además asisten con su propia fuerza de trabajo a su respectiva jornada de molienda, predomina el individualismo frente al uso del bagazo, los compradores, algunas veces frente a precios de venta, y la carencia de vínculos de trabajo colectivo que permitan ver la agroindustria rural, como una agroempresa que podría traer mejores dividendos grupales, innovaciones en procesos, formas de diversificación y mejor inserción al mercado. Estas características dan cuenta entonces de una acción colectiva muy baja.

\section{Descripción de las interacciones}

Por otro lado, el análisis de relaciones de las AIR realizado por medio de talleres participativos, entrevistas con los grupos de productores y la elaboración participativa de las gráficas de red, permitió complementar la dimensión institucional de análisis del SIAL, identificando los vínculos que permiten un flujo de información, cooperación y una confianza entre actores, aproximándose al reflejo de una estructura social donde la AIR es el actor protagónico (Tabla 3). 
Tabla 3. Tipos de relacionamiento estudiados en el SIAL

\begin{tabular}{ll}
\hline Relacionamientos & \multicolumn{1}{c}{ Elementos considerados } \\
\hline Flujo de información & Temas de precios locales y departamentales de la \\
& panela, asuntos técnicos y actualizaciones de la \\
& panela, tendencias y posibilidades del subsector. \\
& La gestión de proyectos, fuentes de financiación y \\
& soporte técnico, logística de entrega y mercadeo de \\
la panela, sugerencias técnicas diversas, recursos & económicos, apoyo financiero en cofinanciación, \\
& apoyos en materia de capacitación y suministro de \\
& insumos, semillas, abonos y equipos. \\
& \\
Expresada en los tipos de acuerdos de pago & flexibles o fiados, almacenamiento de panela \\
vendida, generación de sellos de calidad panelera y & entrega de recursos e insumos sobre la confianza \\
de subuena utilización e implementación.
\end{tabular}

Fuente: Los autores, 2015.

El mapeo participativo de la red de la AIR comunitaria asociada, evidencia una estructura que vincula más actores del orden nacional y gubernamental, no obstante que también predominan actores locales en los procesos de interacción. La destacada bidireccionalidad en los flujos de recursos (información, cooperación, confianza), ayuda a interpretar como una AIR asociada comunitaria y constituida como empresa puede generar mayores vínculos y por ende tener acceso a mayores recursos de la red de actores (Figura 2).

Se pudo constatar en campo con los productores, que el flujo de información entre los actores de esta red, al tener a la AIR como receptor final, ha incidido en el mejoramiento de la capacidad de gestión de esta AIR en el marco del SIAL, por cuanto han logrado identificar diferentes vínculos con el mercado, posibilidades de aplicación a convocatorias de fortalecimiento agroempresarial rural, actualidades del subsector, entre otras.

Adicionalmente, las relaciones de cooperación presentan menos abundancia en reciprocidad del flujo, pasando a tener mayor predominio unidireccional. Esta cooperación se ve materializada en asuntos relacionados con la cofinanciación, apoyos financieros, capacitaciones y apoyo con insumos. Su condición de organización asociativa los ha constituido sin duda como un actor más del municipio, donde han logrado beneficiarse de programas del Ministerio de Agricultura tendientes al fortalecimiento socio-empresarial rural, y diversos programas de capacitación de entidades público-privadas. 


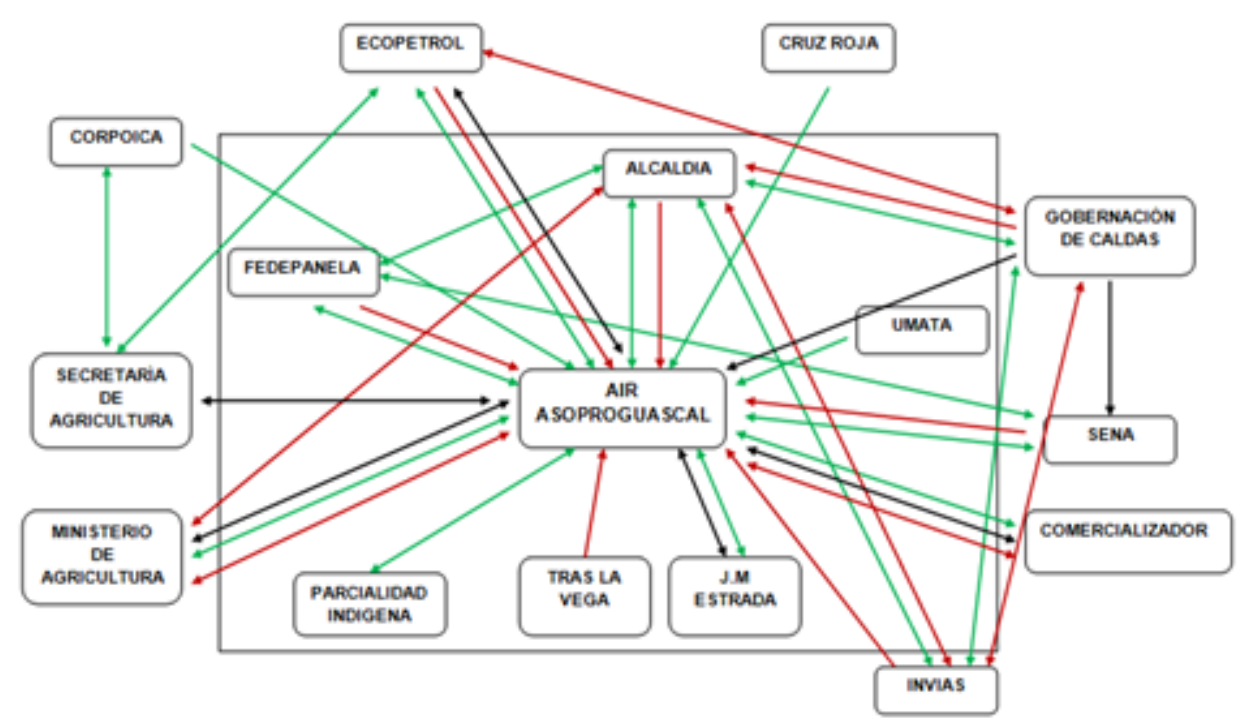

— Flujo de información —— Cooperación Confianza entre actores

Fuente: Los autores, 2015.

Figura 2. Mapa de la red social de la AIR comunitaria asociada

La visualización de relaciones de confianza permite evidenciar menores vínculos entre actores. Esta confianza se refleja por parte de varios actores, básicamente en la entrega de diversos tipos de recursos, construcción de infraestructura, financiamiento, entre otros, sobre la base de sostener su organización asociativa.

Así mismo asuntos como la flexibilidad de pagos con proveedores de insumos, con comercializadores, entre otros, dan cuenta de una relación de confianza que otros actores han tenido en la AIR, algunos de ellos de carácter direccional, recíproco o nulo como el caso de actores locales importantes tales como Fedepanela, como institución representativa panelera y la Unidad Municipal de Asistencia Técnica Agropecuaria (UMATA), como entidad ejecutora de la política agropecuaria. Un actor tan determinante en la dimensión histórica del territorio como lo es el SENA, ha generado procesos de capacitación discontinuos e inconexos, los cuales han terminado por acabar la confianza de parte de la organización de AIR para este caso.

Finalmente, se puede concluir que existen amplios vínculos y formas de relacionamiento tanto con actores locales, pero también del orden departamental y nacional. Así mismo, este esquema de relaciones refleja un caso que le ha permitido a esta AIR comunitaria asociada, lograr diferentes fuentes de financiamiento y apoyo de al menos 5 ó 6 actores de la red, lo cual ha contribuido a su fortalecimiento de su capital humano y social.

Esta aproximación a la identificación de las formas de relacionamiento territorial, puede permitir la planificación de escenarios futuros y de tipo prospectivo para la región. Por ende es necesario avanzar en la caracterización simplificada de los actores que conforman el SIAL, con el fin de identificar alternativas más probables de cohesión y de trabajo conjunto en red (Fontalvo Hererra, Vergara Schmalbach, \& Amézquita López, 2009). 
Por otro lado, dado que la agroindustria rural familiar y artesanal, presenta también una acción colectiva importante, se presenta el mapa de la red social de esta AIR, la cual refleja una estructura más sencilla tanto en número de actores como de vínculos entre ellos. Se evidencia el predominio casi total de los relacionamientos con actores, fundamentalmente del orden local, a través de los cuales se ha favorecido la AIR familiar, tanto en la vereda de estudio como en muchas otras de Supía (Figura 3).
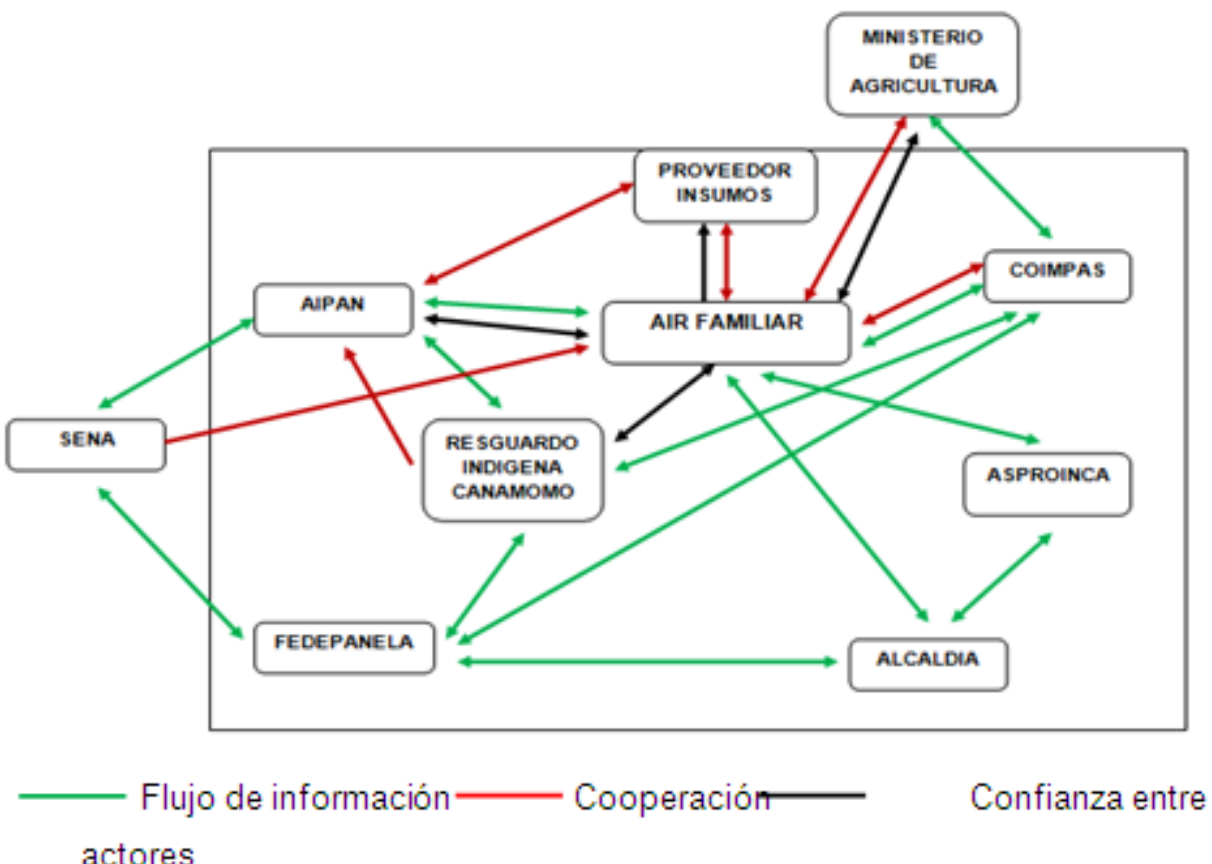

Fuente: Los autores, 2015.

Figura 3. Mapa de la red social de la AIR artesanal y familiar

Con referencia al flujo de información, se destaca que es el vínculo más fluido entre los actores de la red; se nota por primera vez una relevancia de la cooperativa de paneleros "Coimpas" como actor de la red, constituyéndose en su aliado comercial al mantener relaciones más estrechas con este tipo de AIR panelera en Supía.

Este flujo importante de información tiene relación con asuntos técnicos, de manejos de materia prima, higiene de la panela, precios de la panela, información de posibilidades de gestión y financiación de proyectos, novedades del subsector, solo las más destacadas dentro de este vínculo entre actores. Adicionalmente, asuntos como la cofinanciación, el soporte técnico, la proporción de repuestos para la infraestructura, la capacitación y los recursos económicos, son formas de cooperación que fluyen entre los actores de esta red social.

Finalmente, las relaciones de confianza son mínimas comparadas con el análisis de los otros vínculos. En este vínculo la institucionalidad indígena es considerada como la más importante, en donde estos actores representan la mayor centralidad de esta red (resguardo indígena, Asociación Indígena Panelera de Supía - Aipan, Asociación de Productores Indígenas y Campesinos de Riosucio Caldas - Asproinca). Finalmente este tipo de AIR de familiares artesanales, centran sus relacionamientos fundamentalmente con actores locales, facilitándose el acceso a diversos recursos de información y cooperación, pero con amplias dificultades de acceso a mercados 
independientes a la cooperativa y la intermediación, lo que implica estar supeditados a amplias fluctuaciones del precio ante la oferta de bajos volúmenes.

\section{Dimensión alimenticia: diferenciación territorial del producto}

La producción panelera de Supía se ha diferenciado desde el territorio; para ello, la búsqueda del mejoramiento de la confianza con los consumidores ha tenido gran incidencia. De hecho, la ubicación geográfica del municipio ha permitido mejorar la relación productor-consumidor final, a través de una proximidad con vías principales nacionales. Adicionalmente la fiesta de la colación, tradicional desde hace más de 50 años, alrededor de un derivado de la panela, ha contribuido a hacer del territorio una marca con reconocimiento y reputación en la actividad, con clara distinción de la procedencia de los productos y su calidad, que son incluso apetecidos en otros departamentos y ciudades.

Las condiciones agro climáticas de la región han permitido una producción óptima de la caña y a su vez una producción de una panela muy especial, donde en algunas veredas se ha identificado panela altamente apta para pulverizar y acceder a mercados más especializados, situación atribuible a las condiciones y oferta agroclimática así como suelos de la zona.

\section{DISCUSIÓN}

EL territorio panelero de Supía Caldas constituye un SIAL, que cuenta con diversos recursos territoriales (saber-hacer, condiciones locales optimas de clima y suelo para la producción, procesos de innovación tecnológica y organizacional, la reputación del producto), pero también dinámicas (acciones colectivas, formas de relacionamiento local y territorial, conformación de redes), que contribuyen ambas al sostenimiento de la actividad productiva en la región. Los recursos pueden ser o no activados por acciones colectivas destinadas a destacar el territorio y sus componentes como medio para mejorar la eficiencia colectiva de una cadena de producción (Correa Gómez et al., 2006).

El arraigo histórico de un saber-hacer en el territorio (Fournier \& Muchnik, 2012), tanto en las prácticas de cultivo como en el procesamiento agroindustrial, constituye un recurso territorial importante y diferenciador, el cual puede ser visto como una de las formas de innovación del sistema productivo (Wesz Junior, Lovis Trentin, \& Filippi, 2009). Este recurso, de acuerdo con los hallazgos de este estudio, constituye una destacada característica diferenciadora de la región, en especial para pequeños productores, a partir de un saber-hacer que puede generar valor en el producto (Correa Gómez et al., 2006), generando incluso desde la calidad y la procedencia territorial de los productos, una posibilidad más amplia de inserción en los mercados (Wesz Junior et al., 2009).

Dado que el saber-hacer es un recurso territorial específico en la producción panelera de Supía, con una pertenencia histórica y anclaje territorial (Gómez \& Buitrago, 2008; Muchnik, 2006; Zuluaga G., 2001), las formas de transmisión (Corrales Roa \& Forero Álvarez, 2007; Jurado-Alvarán, Buitrago, \& Gómez, 2008; Specht, 2009), han surgido a partir de redes solidarias de cooperación y de intercambios recíprocos entre los productores de Supía, permitiendo el intercambio de saberes y experiencias entre familias y entre miembros de la comunidad, logrando ajustar su conocimiento tanto tácito como explícito (Jurado \& Botero, 2012). 
Los diversos recursos territoriales se han convertido en ventajas para algunas AIR, como organización productiva, la cual mediante la acción colectiva ha logrado transformar recursos en activos territoriales, generando mayores ventajas y potenciamiento para las AIR, logrando adaptarse a un entorno panelero complejo.

La noción de acción colectiva es una dinámica de gran importancia en el análisis de la estructura del SIAL, por cuanto tiene directa relación con la activación de los recursos específicos locales, convirtiendo el territorio en un actor estratégico de competitividad de actividades productivas locales (Specht, 2009). La acción colectiva fue abordada desde la perspectiva de autores como (Boucher, 2006; Correa Gómez et al., 2006; Pomeón et al., 2006; Ramos \& Salcido, 2011; Salas Casasola et al., 2005), los cuales destacaron elementos en común del concepto acción colectiva para el análisis del SIAL, que influyen a su vez en la activación de recursos específicos locales.

Dentro de estos elementos conceptuales y para el análisis de la acción colectiva en la agroindustria rural panelera de Supía, se destacaron: la organización grupal, los relacionamientos y redes horizontales, la confianza, las normas y reglas establecidas, la coordinación horizontal entre actores, la cooperación entre actores, la creación colectiva de capacidades y la generación de estrategias conjuntas de competitividad.

Agroindustrias rurales con dinámicas colectivas más asociativas, tales como las comunitarias asociadas, han logrado retener ese valor agregado para los productores, al insertarse con mayor eficiencia en los mercados, captando mejores precios a través de la innovación en el producto y con mayores volúmenes ofertados, constituyendo a su vez mayores activos específicos del territorio y generando ventajas competitivas.

Del mismo modo, algunas AIR, más que constituir acciones colectivas estructurales fuertes y estables, basan su cooperación horizontal en relaciones familiares y de amistad, como en el caso de algunas AIR comunitarias no asociadas y artesanales individuales, a partir de relacionamientos que pueden generarse desde familias nucleares que se encargan de su propia producción, o a través de formas de asociación entre familias extensas como en el caso de las AIR artesanales familiares donde confluyen diversos parientes con algún grado de consanguinidad (Corrales Roa \& Forero Álvarez, 2007).

Entre tanto, la acción colectiva soportada únicamente en relaciones de amistad, no ha sido contundente en el territorio en materia de generación de valor agregado, inserción a los mercados, estrategias conjuntas o relación colectiva de capacidades. Tal es el caso de la AIR individual artesanal, la cual funciona como una simple unidad de producción, cuya relación de amistad tan solo permite el uso de la infraestructura artesanal.

En ese sentido, los tipos de activos estratégicos del territorio que se pueden originar en el SIAL panelero, han sido analizados por otros autores (Boucher \& Reyes, 2011; Espinosa, 2009; Rodríguez-Borray \& Rangel-Moreno, 2005; Sandoval \& Ruiz, 2005), en los cuales destaca la innovación en producto o en proceso, las capacidades de generar innovaciones organizacionales, la confianza, las estrategias de mercado que permiten eficientes procesos de inserción, la capacidad de cooperación en la AIR que da lugar, por ejemplo, a aprendizajes colectivos, y las redes de relacionamientos generados entre productores, proveedores, transformadores, distribuidores y otros actores.

Otros autores como Boucher \& Reyes (2011), plantean el concepto de economía de las proximidades, como otro activo territorial, a partir de un recurso como la ubicación 
estratégica del municipio y su proximidad geográfica con ciudades y vías nacionales principales, logrando el contacto directo con consumidores desde la panela, y otros derivados y dulcerías como innovaciones en producto, así como el acceso de compradores directamente a las veredas y movilización de los productos a regiones aledañas.

De esta manera algunas agroindustrias rurales, principalmente la AIR comunitaria asociativa y algunas artesanales familiares que han logrado desarrollar un sello de calidad o innovaciones en producto, así como las AIR particulares con gran tecnología, han logrado constituir este activo desde una buena organización y capacidad de cooperación, tanto entre los productores como con otros actores del SIAL, tal es el caso de comercializadores.

\section{CONCLUSIONES}

Los cambios de tipo técnico, social y económico ocurridos en los últimos 50 años, dan cuenta de la existencia en Supía, Caldas, de un SIAL dinámico con raíces históricas y anclaje territorial, con conocimientos transmitidos por varias generaciones, arraigando un saber-hacer que se constituye como uno de los recursos más importantes de la región; al igual que otros recursos territoriales como la tecnología, las condiciones locales de clima y suelo y procesos de innovación.

Se clasificaron en el territorio 5 tipos de AIR, las cuales presentan diferentes formas de interacciones donde se evidencian relacionamientos verticales y horizontales, siendo estos últimos un elemento importante en particular, donde existen acciones colectivas importantes y mayormente dinámicas, tanto en la interacción y organización de la AIR a partir de asignación de roles, formas de aprendizaje mutuo, generación de confianza y el análisis colectivo de estrategias, así como en la interacción con el eslabón de mercado, a partir de la construcción de confianza desde formas más flexibles y cumplidas de pago y niveles de acuerdos de negocio con compradores y proveedores de insumos.

Los recursos locales con que cuenta este territorio, han sido transformados en activos territoriales desde algunas AIR locales con acciones colectivas estructurales y formas de relacionamiento tanto en la propia AIR como en la esfera territorial, como dinámicas que han jugado un rol fundamental para el sostenimiento de la actividad panelera, por medio del mejoramiento de su acceso al mercado con panela de calidad e inocua y con innovación en el proceso y producto a partir del avance en la aplicación y cumplimiento de las condiciones impuestas por la Resolución 779. Tal es el caso de las AIR particulares, comunitarias asociadas, no asociadas y algunas artesanales individuales.

Sin embargo, es importante destacar que si bien es cierto que hoy existe mayor consciencia y cultura colectiva y de la asociatividad en el territorio que en el pasado, aún es determinante estudiar y profundizar en el fomento de relaciones de confianza, cooperación y reciprocidad entre productores locales en el territorio, pues la tendencia para la supervivencia del subsector panelero, pasa por la conformación de agroindustrias rurales comunitarias y asociadas, que logren además de usar los recursos del territorio, una inserción más eficiente en las cadenas de producción y su articulación a redes territoriales donde fluya la información, la cooperación y la confianza entre actores. 
Adicionalmente, sería importante poder estudiar y proponer una hoja de ruta que conlleve a la promoción de una marca con denominación territorial, a partir de los recursos y activos con que se cuenta en la región. Para ello es preciso profundizar en el estudio del desarrollo territorial aplicando el enfoque de sistemas agroalimentarios localizados, como una contribución a que los actores locales se conviertan en agentes activos de su desarrollo mediante la construcción de capacidades e innovación fundamentada en los activos territoriales (Boucher \& González, 2013).

\section{REFERENCIAS}

- Bello, R. (2009). El sistema agroalimentatio localizado (SIAL): otra visión de la lechería de la región centro de Michoacán. Universidad Autónoma Chapingo.

- Boucher, F. (2001). Una visión territorial de la agroindustria rural: Los sistemas agroalimentarios locales. In II Curso Internacional sobre la Promoción de la Agroempresa Rural para el Desarrollo Microregional Sostenible (pp. 1-15). Palmira: CIAT.

- Boucher, F. (2006). Agroindustria Rural y Sistemas Agroalimentarios Locales, nuevos enfoques de desarrollo territorial. In III Congreso Internacional de la Red SIAL "Sistemas Agroalimentarios locales" Alimentación y Territorios "ALTER 2006" (pp. 1-24). Baeza, España: ALTER.

- Boucher, F. (2013). La viabilidad de la agroindustria rural (AIR). El caso de las AIR de la Selva Lacandona, Chiapas, México. Agroalimentaria, 19(36), 71-86.

- Boucher, F., \& González, J. (2013). Guía metodológica para la activación de Sistemas Agroalimentarios Localizados (SIAL).

- Boucher, F., \& Reyes, J. (2011). Guía metodológica para la activación de los SIAL. México. Retrieved from Link

- Castellanos, O., Torres, L., \& Flórez, D. (2010). Agenda prospectiva de investigación y desarrollo tecnológico para la cadena productiva de la panela y su agroindustria en Colombia. Bogotá. Retrieved from Link

- Corrales, E., \& Forero, J. (2007). La reconstrucción de los sistemas de producción campesinos. El caso de Asproinca en Riosucio y Supía. Bogotá: Pontificia Universidad Javeriana. 
- Correa, C., Boucher, F., \& Requier-Desjardins, D. (2006). ¿Cómo "activar" los sistemas agroalimentarios localizados en América Latina? Un análisis comparativo.Agroalimentaria, 22, 17-27.

- Cunha, G. (2012). As convenções sociais de qualidade como suporte à configuração de sistemas agro alimentares locais competitivos: Um estudo Cross Country na pecuaria de corte. Universidade Federal Do Rio Grande Do Sul.

- Denzin, N. , \& Lincoln, Y. (1994). Handbook of qualitative research. Handbook of Qualitative Research (Vol. 1).

- Espinosa, E. (2009). La competitividad del sistema agroalimentario localizado productor de quesos tradicionales. Universidad Autónoma del Estado de México.

- Fontalvo, T., Vergara, J. , \& Amézquita, J. (2009). Construcción de cadenas productivas del sector agrícola mediante modelos de redes: caracterización, simulación de escenarios y prospectiva. Dimensión Empresarial, 7(1), 11-21.

- Fournier, S., \& Muchnik, J. (2012). El enfoque "SIAL" (Sistemas Agroalimentarios Localizados) y la activación de recursos territoriales. Agroalimentaria, 18(34), 133144.

- Gómez, A., \& Buitrago, C. (2008). Condiciones socio-culturales del sector panelero en los municipios de Supía y Riosucio en el alto occidente del departamento de Caldas. Universidad de Caldas.

- IICA. (2009). Una mirada a experiencias exitosas de agroindustria rural en América latina. Instituto Interamericano de Cooperación para la Agricultura: San José, Costa Rica.

- Jurado, C., \& Botero, P. (2012). Trapiche, minga y resistencia. Una experiencia de socialización política. Revista Eleuthera, 7, 167-192.

- Jurado-Alvarán, C., Buitrago, C., \& Gómez, A. (2008). Condiciones socio-culturales del sector panelero en los municipios de Supía y Riosucio en el alto occidente del departamento de Caldas. Manizales.

- Lamnek, S. (2005). Qualitative social research. Berlin: Holz.

- MADR. (2001). Bases para un acuerdo de desarrollo de la cadena agroindustrial de la panela. Instituto Interamericano de Cooperación para la Agricultura. Bogotá: Ministerio de Agricultura y Desarrollo Rural.

- MADR. (2006). El sector panelero colombiano. Retrieved August 22, 2015, fromLink 
- MADR, \& Corporación Colombia Internacional. (2012). Producción de panela en trapiche y precio pagado al productor, 1-10.

- Martín-Crespo, M., \& Salamanca, A. (2007). El muestreo en la investigación cualitativa. Nure Investigación, 27(1), 1-4. Retrieved from Link

- Martínez, H., Ortiz, L., \& Acevedo, X. (2005). La cadena agroindustrial de la panela en Colombia: una mirada global de su estructura y dinámica 1991 - 2005. Bogotá. Retrieved from Link

- Moncayo, E. (2003). Nuevas teorías y enfoques conceptuales sobre el desarrollo regional: ¿hacia un nuevo paradigma? Revista de Economía Institucional, 5(8), 32 65.

- Muchnick, J. (2004). Identidad territorial de los alimentos: alimentar el cuerpo humano y el cuerpo social. In: Territorios y sistemas agroalimentarios locales(pp. 17 - 30). Bogotá: Universidad Nacional de Colombia.

- Muchnik, J. (2006). Sistemas agroalimentarios localizados: evolución del concepto y diversidad de situaciones. In III Congreso Internacional de la Red SIAL “Alimentación y territorios” ALTER 2006 (pp. 1-20). Baeza, España.

- Murdoch, J. (2000). Networks - a new paradigm of rural development? Journal of Rural Studies, 16(4), 407-419. Retrieved from Link

- Noelle-Neumann, E., \& Petersen, T. (2005). Alle, nicht jeder. Einführung in die Methoden der Demoskopie (4a edición). Berlin: Springer.

- Pomeón, T., Boucher, F., Cervantes, F., \& Fournier, S. (2006). Dinámicas colectivas de dos cuencas lecheras mexicanas: Tlaxco, Tlxcala y Tizayuca, Hidalgo. Agroalimentaria, 22, 49-64.

- Pomeón, T., \& Fraire, J. A. (2011). SIAL: un enfoque para el desarrollo territorial. México: REDSIAL.

- Ramos, H., \& Salcido, G. (2011). Sistemas agroalimentarios localizados: el caso de los productores rurales de Nopal en Tlalnepantla, Morelos. México.

- Rodríguez, G., García, H., Roa, Z., \& Santacoloma, P. (2004). Producción de panela como estrategia de diversificación en la generación de ingresos en áreas rurales de América Latina, 81. Retrieved from Link

- Rodríguez-Borray, G., \& Rangel-Moreno, C. (2005). Estudio del sistema agroalimentario localizado, SIAL, de la concentración de fábricas de bocadillo de 
guayaba en las provincias de Vélez y Ricaurte en Colombia. Bogotá: Produmedios.

- Rudas, G., \& Forero, J. (1995). La agroindustria panelera en Colombia. Pequeña producción y relaciones interempresariales. Cuadernos de Desarrollo Rural, (35), 718.

- Salas, I., Boucher, F., \& Requier-Desjardins, D. (2005). Agroindustria rural y liberalización comercial agrícola: el rol de los sistemas agroalimentarios localizados. Agroalimentaria, (22), 29-40.

- Sandoval, N. (2003). Implementación de la metodología Sistemas Agroalimentarios Localizados (SIAL) en la cadena agroindustrial del almidón agrio de yuca en el norte del departamento del Cauca. Universidad Nacional de Colombia.

- Sandoval, V., \& Ruiz, R. (2005). El rol de los recursos locales en la evolución de la agroindustria rural del almidón de yuca en el departamento del Cauca, Colombia.Agroalimentaria, 22, $41-47$.

- Schiffer, E. (2007). Manual: Net-map toolbox influence mapping of social networks. In Sunbelt conference of the international network of social network analysis(pp. 1-19). Corfu, Greece.

- Silva, I. (2003). Disparidades, competitividad territorial y desarrollo local y regional en América Latina. Serie Gestión Pública. Santiago de Chile: CEPAL.

- Simons, H. (2011). El estudio de caso: teoría y práctica. Madrid: Ediciones Morata.

- Specht, S. (2009). O território do morango no vale do Caí - RS: Análise pela perspectiva dos Sistemas Agroalimentares Localizados. Universidade Federal do Rio Grande do Sul.

- Wesz, V., Lovis, I., \& Filippi, E. (2009). Os reflexos das agroindústrias familiares para o desenvolvimento das áreas rurais no Sul do Brasil. Cuadernos de Desarrollo Rural, 6(63), 59-85.

- Yin, R. (2013). Case study research: Design and methods (5a Edición). Los Angeles: Sage publications.

- Zuluaga, A. (2001). Del proceso artesanal agrícola a la agroindustria una visión para el desarrollo rururbano sostenible. Universidad Nacional de Colombia Sede Manizales. 
1. Ingeniero Agrónomo, MSc Desarrollo Rural, Grupo de Investigación GISER Universidad de Antioquia UdeA, Calle 70 No. 52-21, Medellín, Colombia. Estudiante de Doctorado Universidad Nacional de Colombia sede Medellín. email:cjulianmdr@gmail.com

2. Ingeniero Agrícola, MSc, PhD Ciencias Agrícolas, Grupo de Investigación GISER, Profesor Asistente, Facultad de Ciencias Agrarias, Universidad de Antioquia UdeA, Calle 70 No. 52-21, Medellín, Colombia.

Para citar este artículo: Ramírez-Gómez, C.J. y Rodríguez-espinosa, H. (2017) Influencia de los recursos y las dinámicas del territorio en el sostenimiento de la agroindustria rural panelera de Supía, Caldas. Luna Azul, 44, 188-210. DOI: 10.17151/luaz.2017.44.12.

Recuperado de http://200.21.104.25/lunazul/index.php/component/content/article?id=228

Esta obra está bajo una Licencia de Creative Commons Reconocimiento CC BY

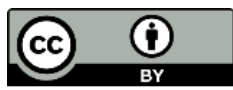

